\title{
NULL CONTROLLABILITY OF NONLOCAL HILFER FRACTIONAL STOCHASTIC DIFFERENTIAL EQUATIONS
}

\author{
JINRONG WANG AND HAMDY M. AHMED
}

Received 08 September, 2017

\begin{abstract}
In this paper, we study exact null controllability of Hilfer fractional semilinear stochastic differential equations in Hilbert spaces. By using fractional calculus and fixed point approach, sufficient conditions of exact null controllability for such fractional systems are established. An example is given to show the application of our results.
\end{abstract}

2010 Mathematics Subject Classification: 26A33; 93B05

Keywords: null controllability, stochastic differential equation, Hilfer fractional derivative

\section{INTRODUCTION}

The stochastic differential equations arise in many mathematical models $[5,14,18$, 20]. The problem of controllability of nonlinear stochastic or deterministic system has been discussed in $[3,4,6,8,9,16,19]$.

Recently, basic theory of differential equations involving Caputo and RiemannLiouville fractional derivatives can be found in $[1,2,13,21-24,26-30]$ and the references cited therein. Beside Caputo and Riemann-Liouville fractional derivatives, there exists a new definition of fractional derivative introduced by Hilfer, which generalized the concept of Riemann-Liouville derivative and has many application in physics, for more details, see [10-12,25].

In this paper, we investigate the exact null controllability of Hilfer fractional semilinear stochastic differential equation of the form

$$
\left\{\begin{array}{l}
D_{0+}^{v, \mu} x(t)=A x(t)+B u(t) \\
+F(t, x(t))+G(t, x(t)) \frac{d \omega(t)}{d t}, t \in J=[0, b], \\
I_{0+}^{(1-v)(1-\mu)} x(0)+h(x)=x_{0},
\end{array}\right.
$$

where $D_{0+}^{v, \mu}$ is the Hilfer fractional derivative, $0 \leq v \leq 1, \frac{1}{2}<\mu<1, A$ is the infinitesimal generator of strongly continuous semigroup of bounded linear operators

This work is supported by National Natural Science Foundation of China (11661016), Training Object of High Level and Innovative Talents of Guizhou Province ((2016)4006), and Unite Foundation of Guizhou Province ([2015]7640), and Graduate ZDKC ([2015]003). 
$S(t), t \geq 0$, on a separable Hilbert space $H$ with inner product $\langle.,$.$\rangle and norm \|$.$\| .$ There exists a $M \geq 1$ such that $\sup _{t \geq 0}\|S(t)\| \leq M$. The control function $u(\cdot)$ is given in $L_{2}(J, U)$, the Hilbert space of admissible control functions with $U$ as a separable Hilbert space. The symbol $B$ stands for a bounded linear operator from $U$ into $H$. Here $\omega$ is an $H$-valued Wiener process associated with a positive, nuclear covariance operator $Q, F$ is an $H$-valued map and $G$ is a $L(K, H)$-valued map both defined on $J \times H$ (where $K$ is a real separable Hilbert space with norn $\|\cdot\|_{K}$ and $L(K, H)$ is the space of all bounded, linear operators from $K$ to $H$, we write simply $L(H)$ if $H=K)$ and $h: C(J, H) \rightarrow H$.

\section{PRELIMINARIES}

In this section, some definitions and results are given which will be used throughout this paper.

Definition 1 (see $[15,17])$. The fractional integral operator of order $\mu>0$ for a function $f$ can be defined as

$$
I^{\mu} f(t)=\frac{1}{\Gamma(\mu)} \int_{0}^{t} \frac{f(s)}{(t-s)^{1-\mu}} d s, t>0
$$

where $\Gamma(\cdot)$ is the Gamma function.

Definition 2 (see [11]). The Hilfer fractional derivative of order $0 \leq v \leq 1$ and $0<\mu<1$ for a function $f$ is defined by

$$
D_{0+}^{\nu, \mu} f(t)=I_{0+}^{\nu(1-\mu)} \frac{d}{d t} I_{0+}^{(1-v)(1-\mu)} f(t) .
$$

Let $(\Omega, \Upsilon, P)$ be a complete probability space furnished with complete family of right continuous increasing sub $\sigma$-algebras $\left\{\Upsilon_{t}: t \in J\right\}$ satisfying $\Upsilon_{t} \subset \Upsilon$. An $\mathrm{H}$ valued random variable is an $\Upsilon$ - measurable function $x(t): \Omega \rightarrow H$ and a collection of random variables $\Psi=\{x(t, \omega): \Omega \rightarrow H \mid t \in J\}$ is called a stochastic process. Usually we suppress the dependence on $\omega \in \Omega$ and write $x(t)$ instead of $x(t, \omega)$ and $x(t): J \rightarrow H$ in the place of $\Psi$. Let $\beta_{n}(t)(n=1,2, \ldots)$ be a sequence of real valued one-dimensional standard Brownian motions mutually independent over $(\Omega, \Upsilon, P)$. Set

$$
\omega(t)=\sum_{n=1}^{\infty} \sqrt{\lambda_{n}} \beta_{n}(t) e_{n}, t \geq 0,
$$

where $\lambda_{n},(n=1,2, \ldots)$ are nonnegative real numbers and $\left\{e_{n}\right\}(n=1,2, \ldots)$ is a complete orthonormal basis in $K$. Let $Q \in L(K, K)$ be an operator defined by $Q e_{n}=$ $\lambda_{n} e_{n}$ with finite $\operatorname{Tr}(Q)=\sum_{n=1}^{\infty} \lambda_{n}<\infty$, (Tr denotes the trace of the operator). Then the above $K$-valued stochastic process $\omega(t)$ is called $Q$-Wiener process.

We assume that $\Upsilon_{t}=\sigma\{\omega(s): 0 \leq s \leq t\}$ is the $\sigma$-algebra generated by $\omega$. 
For $\phi \in L(K, H)$ we define

$$
\|\phi\|_{Q}^{2}=\operatorname{Tr}\left(\phi Q \phi^{*}\right)=\sum_{n=1}^{\infty}\left\|\sqrt{\lambda_{n}} \phi e_{n}\right\|^{2} .
$$

If $\|\phi\|_{Q}^{2}<\infty$, then $\phi$ is called a $Q$-Hilbert-Schmidt operator. Let $L_{Q}(K, H)$ denote the space of all $Q$-Hilbert-Schmidt operators $\phi: K \rightarrow H$. The completion $L_{Q}(K, H)$ of $L(K, H)$ with respect to the topology induced by the norm \|\|$_{Q}$ where $\|\phi\|_{Q}^{2}=\|\phi, \phi\|$ is a Hilbert space with the above norm topology. The collection of all strongly-measurable, square-integrable, $H$-valued random variables, denoted by $L_{2}(\Omega, H)$, is a Banach space equipped with norm $\|x(\cdot)\|_{L_{2}(\Omega, H)}=$ $\left(E\|x(., \omega)\|^{2}\right)^{\frac{1}{2}}$, where the expectation, $E$ is defined by $E(x)=\int_{\Omega} x(\omega) d P$. An important subspace of $L_{2}(\Omega, H)$ is given by $L_{2}^{0}(\Omega, H)=\left\{x \in L_{2}(\Omega, H), x\right.$ is $\Upsilon_{0^{-}}$measurable $\}$.

Let $C\left(J, L_{2}(\Omega, H)\right)$ be the Banach space of all continuous maps from $J$ into $L_{2}(\Omega, H)$ satisfying the condition $\sup _{t \in J} E\|x(t)\|^{2}<\infty$.

Define $Y=\left\{x: t^{(1-v)(1-\mu)} x(t) \in C\left(J, L_{2}(\Omega, H)\right)\right\}$, with norm $\|\cdot\|_{Y}$ defined by $\|\cdot\|_{Y}=\left(\sup _{t \in J} E\left\|t^{(1-v)(1-\mu)} x(t)\right\|^{2}\right)^{\frac{1}{2}}$. Obviously, $Y$ is a Banach space.

For $x \in H$, we define two families of operators $\left\{S_{\nu, \mu}(t): t \geq 0\right\}$ and $\left\{P_{\mu}(t): t \geq 0\right\}$ by

$$
S_{v, \mu}(t)=I_{0+}^{\nu(1-\mu)} P_{\mu}(t), P_{\mu}(t)=t^{\mu-1} T_{\mu}(t), T_{\mu}(t)=\int_{0}^{\infty} \mu \theta \Psi_{\mu}(\theta) S\left(t^{\mu} \theta\right) d \theta,
$$

where

$$
\Psi_{\mu}(\theta)=\sum_{n=1}^{\infty} \frac{(-\theta)^{n-1}}{(n-1) ! \Gamma(1-n \mu)}, 0<\mu<1, \theta \in(0, \infty)
$$

is a function of Wright-type which satisfies

$$
\int_{0}^{\infty} \theta^{\Psi} \Psi_{\mu}(\theta) d \theta=\frac{\Gamma(1+\Psi)}{\Gamma(1+\mu \Psi)}
$$

for $\theta \geq 0$.

Lemma 1 (see [10]). The operator $S_{v, \mu}$ and $P_{\mu}$ have the following properties.

(i) $\left\{P_{\mu}(t): t>0\right\}$ is continuous in the uniform operator topology.

(ii) For any fixed $t>0, S_{v, \mu}(t)$ and $P_{\mu}(t)$ are linear and bounded operators, and

$$
\left\|P_{\mu}(t) x\right\| \leq \frac{M t^{\mu-1}}{\Gamma(\mu)}\|x\|,\left\|S_{v, \mu}(t) x\right\| \leq \frac{M t^{(v-1)(\mu-1)}}{\Gamma(v(1-\mu)+\mu)}\|x\| .
$$

(iii) $\left\{P_{\mu}(t): t>0\right\}$ and $\left\{S_{v, \mu}(t): t>0\right\}$ are strongly continuous. 
To study the exact null controllability of (1.1) we consider the fractional linear system

$$
\left\{\begin{array}{l}
D_{0+}^{v, \mu} y(t)=A y(t)+B u(t)+F(t)+G(t) \frac{d \omega(t)}{d t}, t \in J=[0, b], \\
I_{0+}^{(1-v)(1-\mu)} y(0)=y_{0}
\end{array}\right.
$$

associated with the system (1.1).

Define the operator

$$
L_{0}^{b} u=\int_{0}^{b} P_{\mu}(b-s) B u(s) d s: L_{2}(J, U) \rightarrow H,
$$

where $L_{0}^{b} u$ has a bounded inverse operator $\left(L_{0}\right)^{-1}$ with values in $L_{2}(J, U) / \operatorname{ker}\left(L_{0}^{b}\right)$, and

$$
\begin{aligned}
N_{0}^{b}(y, F, G) & =S_{\nu, \mu}(b) y+\int_{0}^{b} P_{\mu}(b-s) F(s) d s \\
& +\int_{0}^{b} P_{\mu}(b-s) G(s) d \omega(s): H \times L_{2}(J, U) \rightarrow H .
\end{aligned}
$$

Definition 3. The system (2.1) is said to be exactly null controllable on $J$ if

$$
\operatorname{Im} L_{0}^{b} \supset \operatorname{Im} N_{0}^{b} .
$$

By [7], the system (2.1) is exactly null controllable if there exists $\gamma>0$ such that

$$
\left\|\left(L_{0}^{b}\right)^{*} y\right\|^{2} \geq \gamma\left\|\left(N_{0}^{b}\right)^{*} y\right\|^{2}
$$

for all $y \in H$.

Lemma 2 (see [16]). Suppose that the linear system (2.1) is exactly null controllable on $J$. Then the linear operator

$$
W=\left(L_{0}\right)^{-1} N_{0}^{b}: H \times L_{2}(J, H) \rightarrow L_{2}(J, U)
$$

is bounded and the control

$$
\begin{aligned}
u(t) & =-\left(L_{0}\right)^{-1}\left[S_{v, \mu}(b) y_{0}+\int_{0}^{b} P_{\mu}(b-s) F(s) d s+\int_{0}^{b} P_{\mu}(b-s) G(s) d \omega(s)\right] \\
& =-W\left(y_{0}, F, G\right)
\end{aligned}
$$

transfers the system (2.1) from $y_{0}$ to 0 , where $L_{0}$ is the restriction of $L_{0}^{b}$ to $\left[\operatorname{ker} L_{0}^{b}\right]^{\perp}$, $F \in L_{2}(J, H)$ and $G \in L_{2}(J, L(K, H))$. 


\section{EXACT NULL CONTROLLABILITY}

In this section, we formulate sufficient conditions for exact null controllability for the system (1.1). First, we give the definitions of mild solution and exact null controllability for it.

Definition 4. We say $x \in C\left(J, L_{2}(\Omega, H)\right)$ is a mild solution to (1.1) if it satisfies that

$$
\begin{aligned}
x(t) & =S_{v, \mu}(t)\left[x_{0}-h(0)\right]+\int_{0}^{t} P_{\mu}(t-s)[F(s, x(s))+B u(s)] d s \\
& +\int_{0}^{t} P_{\mu}(t-s) G(s, x(s)) d \omega(s), t \in J .
\end{aligned}
$$

Definition 5. The system (1.1) is said to be exact null controllable on the interval $J$ if there exists a stochastic control $u \in L_{2}(J, U)$ such that the solution $x$ of the system (1.1) satisfies $x(b)=0$.

To prove the main result, we need the following hypotheses:

(H1) The fractional linear system (2.1) is exactly null controllable on $J$.

(H2) The function $F: J \times H \rightarrow H$ is locally Lipschitz continuous, for all $t \in$ $J, x, x_{1}, x_{2} \in H$, there exist constant $c_{1}>0$, such that

$$
\left\|F\left(t, x_{2}\right)-F\left(t, x_{1}\right)\right\|^{2} \leq c_{1}\left\|x_{2}-x_{1}\right\|^{2}, \quad\|F(t, x)\|^{2} \leq c_{1}\left(1+\|x\|^{2}\right) .
$$

(H3) The function $G: J \times H \rightarrow L(K, H)$ is locally Lipschitz continuous, for all $t \in J, x, x_{1}, x_{2} \in H$, there exist constant $c_{2}>0$, such that

$$
\left\|G\left(t, x_{2}\right)-G\left(t, x_{1}\right)\right\|_{Q}^{2} \leq c_{2}\left\|x_{2}-x_{1}\right\|^{2}, \quad\|G(t, x)\|_{Q}^{2} \leq c_{2}\left(1+\|x\|^{2}\right) .
$$

$(H 4)$ The function $h: C(J, H) \rightarrow H$ is continuous, for any $x, x_{1}, x_{2} \in C(J, H)$, there exist constant $c_{3}>0$, such that

$$
\begin{gathered}
\left\|h\left(x_{2}\right)-h\left(x_{1}\right)\right\|^{2} \leq c_{3}\left\|x_{2}-x_{1}\right\|^{2}, \quad\|h(x)\|^{2} \leq c_{3}\left(1+\|x\|^{2}\right) \\
\text { Set } \quad \varrho_{1}:=\frac{4 M^{2} c_{3}}{\Gamma^{2}(v(1-\mu)+\mu)}+\frac{M^{2} b^{1+2 v(\mu-1)}}{(2 \mu-1) \Gamma^{2}(\mu)}\left(c_{1}+c_{2} \operatorname{Tr}(Q)\right) \quad \text { and } \\
\varrho_{2}:=1+\frac{4 M^{2} b^{2 \mu-1}\|W\|^{2}\|B\|^{2}}{(2 \mu-1) \Gamma^{2}(\mu)}
\end{gathered}
$$

Theorem 1. If the hypotheses $(H 1)-(H 4)$ are satisfied, then the system (1.1) is exactly null controllable on $J$ provided that

$$
\varrho:=\varrho_{1} \varrho_{2}<1 .
$$

Proof. For an arbitrary $x$ define the operator $\Phi$ on $Y$ as follows

$$
\begin{aligned}
(\Phi x)(t) & =S_{v, \mu}(t)\left[x_{0}-h(x)\right] \\
& +\int_{0}^{t} P_{\mu}(t-s)\left[F(s, x(s))-B W\left(x_{0}-h(x), F, G\right)\right] d s
\end{aligned}
$$




$$
+\int_{0}^{t} P_{\mu}(t-s) G(s, x(s)) d \omega(s), t \in J,
$$

where

$$
\begin{aligned}
u(t) & =W\left(x_{0}-h(x), F, G\right)(t) \\
& =-\left(L_{0}\right)^{-1}\left\{S_{v, \mu}(b)\left[x_{0}-h(x)\right]\right. \\
& \left.+\int_{0}^{b} P_{\mu}(b-s) F(s, x(s)) d s+\int_{0}^{b} P_{\mu}(b-s) G(s, x(s)) d \omega(s)\right\} .
\end{aligned}
$$

It will be shown that the operator $\Phi$ from $Y$ into itself has a fixed point.

Step 1. The control $u(\cdot)=-W\left(x_{0}-h(x), F, G\right)$ is bounded on $Y$.

Indeed,

$$
\begin{aligned}
\|u\|_{Y}^{2} & =\sup _{t \in J} t^{2(1-v)(1-\mu)} E\|u\|^{2} \\
& \leq \sup _{t \in J} t^{2(1-v)(1-\mu)} E\left\|W\left(x_{0}-h(x), F, G\right)(s)\right\|^{2} \\
& \leq\|W\|^{2}\left\{\frac{M^{2}}{\Gamma^{2}(v(1-\mu)+\mu)}\left[E\left\|x_{0}\right\|^{2}+c_{3}\left(1+E\|x\|^{2}\right)\right]\right. \\
& \left.+\frac{M^{2} b^{1+2 v(\mu-1)}}{(2 \mu-1) \Gamma^{2}(\mu)}\left(1+E\|x\|^{2}\right)\left(c_{1}+c_{2} \operatorname{Tr}(Q)\right)\right\} .
\end{aligned}
$$

Step 2. We show that $\Phi$ maps $Y$ into itself.

From (3.2) and (3.3) for $t \in J$, we have

$$
\begin{aligned}
\|(\Phi x)(t)\|_{Y}^{2} & =\sup _{t \in J} t^{2(1-v)(1-\mu)} E\|(\Phi x)(t)\|^{2} \\
& \leq 4 \sup _{t \in J} t^{2(1-v)(1-\mu)}\left\{E\left\|S_{v, \mu}(t)\left[x_{0}-h(x)\right]\right\|^{2}\right. \\
& \left.+E\left\|\int_{0}^{t} P_{\mu}(t-s) F(s, x(s)) d s\right\|^{2}\right\} \\
& +4 \sup _{t \in J} t^{2(1-v)(1-\mu)} \\
& \times\left\{E\left\|\int_{0}^{t} P_{\mu}(t-s)\left[B W\left(x_{0}-h(x), F, G\right)(s)\right] d s\right\|^{2}\right. \\
& \left.+E\left\|\int_{0}^{t} P_{\mu}(t-s) G(s, x(s)) d \omega(s)\right\|^{2}\right\} \\
& \leq\left[\frac{4 M^{2}}{\Gamma^{2}(v(1-\mu)+\mu)}\left[E\left\|x_{0}\right\|^{2}+c_{3}\left(1+E\|x\|^{2}\right)\right]\right.
\end{aligned}
$$




$$
\begin{aligned}
& \left.+\frac{4 M^{2} b^{1+2 v(\mu-1)}}{(2 \mu-1) \Gamma^{2}(\mu)}\left(1+E\|x\|^{2}\right)\left(c_{1}+c_{2} \operatorname{Tr}(Q)\right)\right] \\
& \times\left[1+\frac{M^{2}\|B\|^{2}\|W\|^{2} \| b^{2 \mu-1}}{(2 \mu-1) \Gamma^{2}(\mu)}\right]<\infty .
\end{aligned}
$$

Therefore $\Phi$ maps $Y$ into itself.

Step 3. We prove $(\Phi x)(t)$ is continuous on $J$ for any $x \in Y$.

Let $0<t \leq b$ and $\epsilon>0$ be sufficiently small, then,

$$
\begin{aligned}
& \|(\Phi x)(t+\epsilon)-(\Phi x)(t)\|_{Y}^{2} \\
& =\sup _{t \in J} t^{2(1-v)(1-\mu)} E\|(\Phi x)(t+\epsilon)-(\Phi x)(t)\|^{2} \\
& \leq 4 \sup _{t \in J} t^{2(1-v)(1-\mu)} \\
& \times E\left\|\left(S_{v, \mu}(t+\epsilon)-S_{v, \mu}(t)\right)\left[x_{0}-h(x)\right]\right\|^{2} \\
& +4 \sup _{t \in J} t^{2(1-v)(1-\mu)} \\
& \times E \| \int_{0}^{t+\epsilon} P_{\mu}(t+\epsilon-s)\left[B W\left(x_{0}-h(x), F, G\right)(s)\right] d s \\
& -\int_{0}^{t} P_{\mu}(t-s)\left[B W\left(x_{0}-h(x), F, G\right)(s)\right] d s \|^{2} \\
& +4 \sup _{t \in J} t^{2(1-v)(1-\mu)} \\
& \times E\left\|\int_{0}^{t+\epsilon} P_{\mu}(t+\epsilon-s) F(s, x(s)) d s-\int_{0}^{t} P_{\mu}(t-s) F(s, x(s)) d s\right\|^{2} \\
& +4 \sup _{t \in J} t^{2(1-v)(1-\mu)} \\
& \times E\left\|\int_{0}^{t+\epsilon} P_{\mu}(t+\epsilon-s) G(s, x(s)) d \omega(s)-\int_{0}^{t} P_{\mu}(t-s) G(s, x(s)) d \omega(s)\right\|^{2} .
\end{aligned}
$$

Clearly, from Lemma 1, (H2) and (H3), the right hand side of (3.4) tends to zero as $\epsilon \rightarrow 0$. Hence, $(\Phi x)(t)$ is continuous on $J$.

Step 4. We show that $(\Phi x)(t)$ is a contraction on $Y$.

Let $x_{1}, x_{2} \in Y$, for any $t \in(0, b]$ be fixed, then

$$
\begin{aligned}
& \left\|\left(\Phi x_{2}\right)(t)-\left(\Phi x_{1}\right)(t)\right\|_{Y}^{2} \\
& =\sup _{t \in J} t^{2(1-v)(1-\mu)} E\left\|\left(\Phi x_{2}\right)(t)-\left(\Phi x_{1}\right)(t)\right\|^{2} \\
& \leq 4 \sup _{t \in J} t^{2(1-v)(1-\mu)} E \|\left(S_{v, \mu}(t)\left[h\left(x_{2}\right)-h\left(x_{1}\right)\right] \|^{2}\right.
\end{aligned}
$$




$$
\begin{aligned}
& +4 \sup _{t \in J} t^{2(1-v)(1-\mu)} E \| \int_{0}^{t} P_{\mu}(t-s)\left[B W\left(x_{0}-h\left(x_{2}\right), F, G\right)(s)\right. \\
& \left.-B W\left(x_{0}-h\left(x_{1}\right), F, G\right)(s)\right] d s \|^{2} \\
& +4 \sup _{t \in J} t^{2(1-v)(1-\mu)} E\left\|\int_{0}^{t} P_{\mu}(t-s)\left[F\left(s, x_{2}(s)\right)-F\left(s, x_{1}(s)\right)\right] d s\right\|^{2} \\
& +4 \sup _{t \in J} t^{2(1-v)(1-\mu)} E\left\|\int_{0}^{t} P_{\mu}(t-s)\left[G\left(s, x_{2}(s)\right)-G\left(s, x_{1}(s)\right)\right] d \omega(s)\right\|^{2} \\
& \leq \varrho E\left\|x_{2}-x_{1}\right\|^{2} .
\end{aligned}
$$

Hence, $\Phi$ is a contraction in $Y$ via (3.1). From the Banach fixed point theorem, $\Phi$ has a unique fixed point. Therefore the system (1.1) is exact null controllable on $J$.

\section{AN EXAMPLE}

Consider the following Hilfer fractional stochastic partial differential system

$$
\left\{\begin{array}{l}
D_{0+}^{v, \frac{2}{3}} x(t, z)=\frac{\partial^{2}}{\partial z^{2}} x(t, z)+u(t, z) \\
+f(t, x(t, z))+g(t, x(t, z)) \frac{d \omega(t)}{d t}, t \in J, 0<z<1, \\
x(t, 0)=x(t, 1)=0, t \in J, \\
I_{0+}^{\frac{1}{3}(1-v)}(x(0, z))+\sum_{i=1}^{p} k_{i} x\left(t_{i}, z\right)=x_{0}(z), 0 \leq z \leq 1,
\end{array}\right.
$$

where $p$ is a positive integer, $0<t_{0}<t_{1}<\ldots<t_{p}<b$ and $\omega(t)$ is Wiener process, $u \in L_{2}(0, b)$, and $H=L_{2}([0,1])$. Let $f: R \times R \rightarrow R$ and $g: R \times R \rightarrow R$ are continuous and global Lipschitz continuous in the second variable. Also, let $A: H \rightarrow H$ be defined by $A y=\frac{\partial^{2}}{\partial z^{2}} y$ with domain $D(A)=\left\{y \in H: y, \frac{\partial y}{\partial z}\right.$ are absolutely continuous, and $\frac{\partial^{2} y}{\partial z^{2}} \in H, y(0)=y(1)=0$.

It is known that $A$ is self-adjoin and has the eigenvalues $\lambda_{n}=-n^{2} \pi^{2}, n \in N$, with the corresponding normalized eigenvectors $e_{n}(z)=\sqrt{2} \sin (n \pi z)$. Furthermore, $A$ generates an analytic compact semigroup of bounded linear operator $S(t), t \geq 0$, on a separable Hilbert space $H$ which is given by

$$
S(t) y=\sum_{n=1}^{\infty}\left(y_{n}, e_{n}\right) e_{n}=\sum_{n=1}^{\infty} 2 e^{-n^{2} \pi^{2} t} \sin (n \pi z) \int_{0}^{1} \sin (n \pi \xi) y(\xi) d \xi, y \in H .
$$

If $u \in L_{2}(J, H)$, then $B=I, B^{*}=I$. 
Now we consider

$$
\left\{\begin{array}{l}
D_{0+}^{v, \frac{2}{3}} y(t, z)=\frac{\partial^{2}}{\partial z^{2}} y(t, z)+u(t, z) \\
+f(t, z)+g(t, z) d \omega(t), \quad t \in J, 0<z<1, \\
y(t, 0)=y(t, 1)=0, t \in J \\
I_{0+}^{\frac{1}{3}(1-v)}(y(0, z))=y_{0}(z), 0 \leq z \leq 1
\end{array}\right.
$$

The system (4.2) is exact null controllability if there is a $\gamma>0$, such that

$$
\int_{0}^{b}\left\|B^{*} P_{\mu}^{*}(b-s) y\right\|^{2} d s \geq \gamma\left[\left\|S_{\mu, \nu}^{*}(b) y\right\|^{2}+\int_{0}^{b}\left\|P_{\mu}^{*}(b-s) y\right\|^{2} d s\right],
$$

or equivalently

$$
\int_{0}^{b}\left\|P_{\mu}(b-s) y\right\|^{2} d s \geq \gamma\left[\left\|S_{\mu, \nu}(b) y\right\|^{2}+\int_{0}^{b}\left\|P_{\mu}(b-s) y\right\|^{2} d s\right] .
$$

If $f=0$ and $g=0$ in (4.2), then the fractional linear system is exactly null controllable if

$$
\int_{0}^{b}\left\|P_{\mu}(b-s) y\right\|^{2} d s \geq b\left\|S_{\mu, \nu}(b) y\right\|^{2} .
$$

Therefore,

$$
\int_{0}^{b}\left\|P_{\mu}(b-s) y\right\|^{2} d s \geq \frac{b}{1+b}\left[\left\|S_{\mu, v}(b) y\right\|^{2}+\int_{0}^{b}\left\|P_{\mu}(b-s) y\right\|^{2} d s\right] .
$$

Hence, the linear fractional system (4.2) is exactly null controllable on $[0, b]$. So the hypothesis $\left(H_{1}\right)$ is satisfied.

We define $F: J \times H \rightarrow H, G: J \times H \rightarrow L(K, H)$ and $h: C(J, H) \rightarrow H$ as follows: $F(t, x)=f(t, x(t, z)), G(t, x)=g(t, x(t, z))$ and $h(x)=\sum_{i=1}^{p} k_{i} x\left(t_{i}, z\right)$ Then $h(\cdot)$ satisfies $(H 4)$.

By choosing the constants $k_{i}, i=1,2, \ldots, p, M, c_{1}, c_{2}$ and $c_{3}$ such that $\varrho<$ 1. Hence, all the hypotheses of Theorem 1 are satisfied, so the Hilfer fractional stochastic partial differential system (4.1) is exact null controllable on $[0, b]$.

\section{REFERENCES}

[1] R. Agarwal, S. Hristova, and D. O'Regan, "A survey of Lyapunov functions, stability and impulsive Caputo fractional differential equations," Fract. Calc. Appl. Anal, vol. 19, no. 2, pp. 290-318, 2016, doi: 10.1515/fca-2016-0017.

[2] R. P. Agarwal, M. Benchohra, and S. Hamani, "A survey on existence results for boundary value problems of nonlinear fractional differential equations and inclusions," Acta Appl. Math, vol. 109, no. 3, pp. 973-1033, 2010, doi: 10.1007/s10440-008-9356-6.

[3] H. M. Ahmed, "Controllability of fractional stochastic delay equations," Lobachevskii J. Math, vol. 30, no. 3, pp. 195-202, 2009, doi: 10.1134/S19950802090300019. 
[4] H. M. Ahmed, "Approximate controllability of impulsive neutral stochastic differential equations with fractional Brownian motion in a Hilbert space," Adv. Difference Equ, vol. 2014, no. 113, pp. 1-11, 2014, doi: 10.1186/1687-1847-2014-113.

[5] K. Balachandran, P. Balasubramaniam, and J. P. Dauer, "Local null controllability of nonilinear functional differential systems in Banach spaces," J. Optim Theory Appl., vol. 88, no. 1, pp. 61-75, 1996, doi: 0022-3239/96/0100.0061509,50/.

[6] K. Balachandran and J. H. Kim, "Sample controllability of nonlinear stochastic integrodifferential systems," Nonlinear Anal.: Hybrid Syst, vol. 4, no. 3, pp. 543-549, 2010, doi: 10.1016/j.nahs.2010.02.001.

[7] R. F. Curtain and H. Zwart, An Introduction to Infinite Dimensional Linear Systems Theory. New York: World Scientific, 1995. doi: 10.1007/978-1-4612-4224-6.

[8] J. P. Dauer and P. Balasubramaniam, "Null controllability of semilinear integrodifferential systems in Banach space," Appl. Math. Lett, vol. 10, no. 6, pp. 117-123, 1997, doi: 10.1016/S08939659(97)00114-6.

[9] X. Fu and Y. Zhang, "Exact null controllability of non-autonomous functional evolution systems with nonlocal conditions," Acta Math. Sci, vol. 33B, no. 3, pp. 747-757, 2013, doi: 10.1016/S0252-9602(13)60035-1.

[10] H. B. Gu and J. J. Trujillo, "Existence of mild solution for evolution equation with Hilfer fractional derivative," Appl. Math. Comput, vol. 257, pp. 344-354, 2015, doi: 10.1016/j.amc.2014.10.083.

[11] R. Hilfer, Applications of Fractional Calculus in Physics. Singapore: World Scientific, 2000.

[12] R. Hilfer, "Experimental evidence for fractional time evolution in glass materials," Chem. Phys, vol. 284, no. 1-2, pp. 399-408, 2002, doi: org/10.1016/S0301-0104(02)00670-5.

[13] J. W. M. Li, "Finite time stability of fractional delay differential equations," Appl. Math. Lett, vol. 64, no. 3, pp. 170-176, 2017, doi: org 10.1016/j.aml.2016.09.004.

[14] X. Mao, Stochastic differential equations and applications. Horwood: Chichester, 1997.

[15] K. S. Miller and B. Ross, An Introduction to the fractional calculus and fractional differential equations. New York: John Wiley, 1993.

[16] J. Y. Park and P. Balasubramaniam, "Exact null controllability of abstract semilinear functional integrodifferential stochastic evolution equations in Hilbert spaces," Taiwanese J. Math, vol. 13, no. 6B, pp. 2093-2103, 2009.

[17] I. Podlubny, Fractional differential equations. San Diego: Academic press, 1999.

[18] P. Protter, Stochastic integration and differential equations, Applications of Mathematics. New York: Springer, Berlin, 1990. doi: 10.1007/978-3-662-02619-9.

[19] R. Sakthivel, S. Suganya, and S. M. Anthoni, "Approximate controllability of fractional stochastic evolution equations," Comput. Math. Appl, vol. 63, no. 3, pp. 660-668, 2012, doi: org/10.1016/j.camwa.2011.11.024.

[20] K. Sobczyk, Stochastic differential equations with applications to Physics and Engineering. London: Kluwer Academic, 1991. doi: 10.1007/978-94-011-3712-6.

[21] J. Wang, "Approximate mild solutions of fractional stochastic evolution equations in Hilbert spaces," Appl. Math. Comput, vol. 256, pp. 315-323, 2015, doi: 10.1016/j.amc.2014.12.155.

[22] J. Wang, A. G. Ibrahim, and M. Fečkan, "Differential inclusions of arbitrary fractional order with anti-periodic conditions in Banach spaces," Electron. J. Qual. Theory Differ. Equ, vol. 2016, no. 34, pp. 1-22, 2016, doi: 10.14232/ejqtde.2016.1.34.

[23] J. Wang and X. Li, "A Uniformed Method to Ulam-Hyers Stability for Some Linear Fractional Equations," Mediterr. J. Math, vol. 13, no. 2, pp. 625-635, 2016, doi: org/10.1007/s00009-0150523-5.

[24] J. Wang, X. Li, M. Fečkan, and Y. Zhou, "Hermite-Hadamard-type inequalities for RiemannLiouville fractional integrals via two kinds of convexity," Appl. Anal, vol. 92, no. 11, pp. 22412253, 2013, doi: 10.1080/00036811.2012.727986. 
[25] J. Wang and Y. Zhang, "Nonlocal initial value problems for differential equations with Hilfer fractional derivative," Appl. Math. Comput, vol. 266, no. 1, pp. 850-859, 2015, doi: org/10.1016/j.amc.2015.05.144.

[26] J. Wang, Y. Zhou, and Z. Lin, "On a new class of impulsive fractional differential equations," Appl. Math. Comput, vol. 242, no. 1, pp. 649-657, 2014, doi: org/10.1016/j.amc.2014.06.002.

[27] Y. Zhou, J. R. Wang, and L. Zhang, Basic Theory of Fractional Differential Equations. Singapore: World Scientific, 2016.

[28] Y. Zhou and L. Zhang, "Existence and multiplicity results of homoclinic solutions for fractional Hamiltonian systems," Comput. Math. Appl, vol. 73, no. 6, pp. 1325-1345, 2017, doi: org/10.1016/j.camwa.2016.04.041.

[29] Y. Zhou, L. Zhang, and X. H. Shen, "Existence of mild solutions for fractional evolution equations," J. Int. Equ. Appl, vol. 25, no. 4, pp. 557-586, 2013, doi: 10.1216/JIE-2013-25-4-557.

[30] Y. Zhou, F. Zhou, and J. Pecaric, "Abstract Cauchy problem for fractional functional differential equations," Topol. Meth. Nonlinear Anal, vol. 42, no. 1, pp. 119-136, 2013, doi: projecteuclid.org/euclid.tmna/1461247296.

Authors' addresses

JinRong Wang

Guizhou University, Department of Mathematics, Guiyang 550025, China

E-mail address: sci.jrwangegzu.edu.cn

\section{Hamdy M. Ahmed}

Higher Institute of Engineering, El-Shorouk Academy, El-Shorouk City, Cairo, Egypt

E-mail address: hamdy_17eg@yahoo.com 